\title{
A SIMULATIVE APPROACH TO ELECTRON CONDUCTION IN THICK-FILM RESISTORS
}

\author{
C. JACOBONI, M. PRUDENZIATI and A. RIZZI \\ Istituto di Fisica dell' Universitd di Modena, via Campi 213/A, 41100 Modena, Italy
}

(Received November 13, 1981)

\begin{abstract}
A simulative approach to the calculation of electrical transport in thick-film resistors is presented, in which electrons are considered to hop from and to metallic grains and localized states in the glass. For concentrations of metallic grains sufficiently low and of localized states sufficiently high, a maximum in conductivity as a function of temperature is obtained due to a balance between the tendency of temperature to favour hopping and to oppose an ordered response to an external force.
\end{abstract}

\section{INTRODUCTION}

The technological importance of thick films needs not be emphasized here. They present, however, some interesting problems also from the point of view of basic physics, related in particular to their electrical transport properties, which are not yet totally understood. ${ }^{1}$ An important example is the maximum shown by the conductivity as a function of temperature, indicated in Figure 1, which is a general property of most thick-film resistors (TFR), but still waits for a definite, convincing, explanation.

The complexity of the morphological nature of TFRs is such that it is difficult to formulate adequate theoretical models, and when such models are someway obtained, they are never simple enough to yield exact analytical solutions for their transport properties; analytical approximations are usually necessary as to make any result obtained questionable.

For these reasons a project has been undertaken of computer simulation of TFRs which aims at formulating models which are simple enough for their computer simulation and obtaning, by means of Monte Carlo calculations, the transport properties predicted by the model without any further approximations, besides those inherent in the model itself. The complexity of the model may eventually be increased, and further details of physical thick films may be introduced into it without particular difficulties.

\section{THE MODEL}

In this first simulative approach to the problem only the essential features of TFRs are introduced into the model: non-overlapping metal spheres (grains) are randomly embedded in a glassy matrix which contains localized electron states randomly distributed in space $\vec{R}$ and energy $\mathrm{E}\left(\mathrm{E}_{1}<\mathrm{E}<\mathrm{E}_{2}\right)$ with uniform distributions, as shown in Figure 2.

Electrons may jump from localized states or grains to other localized states or grains. When an external voltage is applied, jumps along the direction of the electric force will be favoured, thus producing a net current. However, if we try to introduce an external voltage in the simulation, we have to solve the difficult problem of the field distribution 


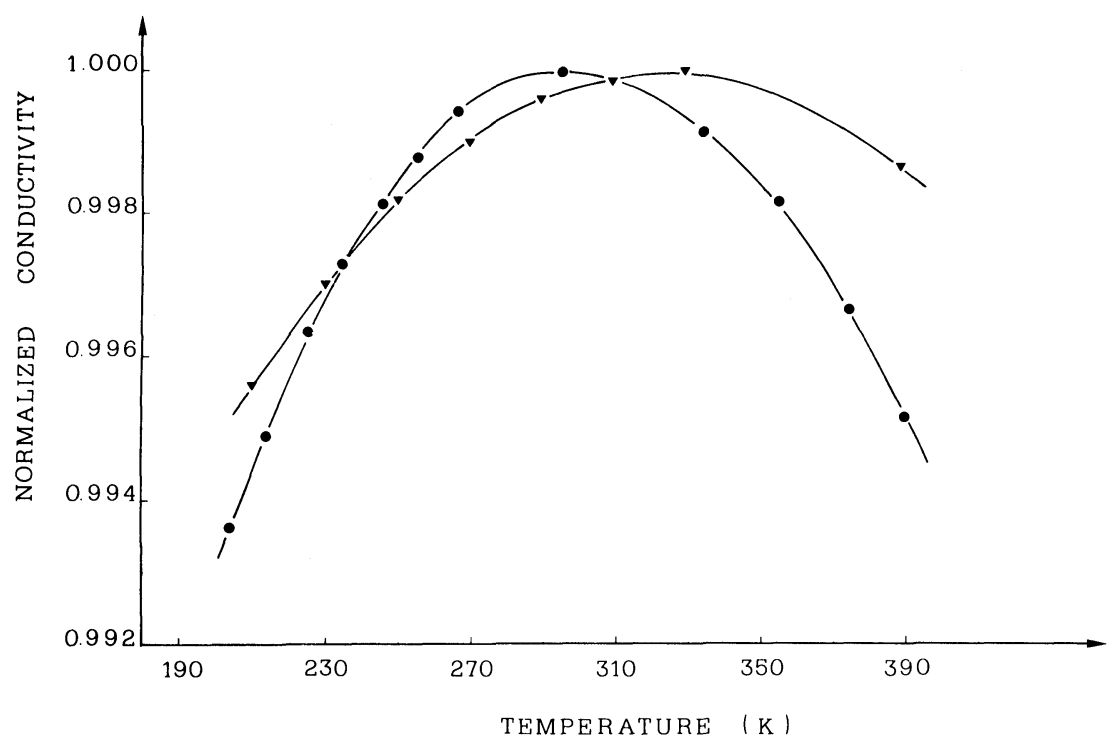

FIGURE 1 Conductivity of two thick-film resistors as a function of temperature. $\bullet$ Rutheniumpyrochlore-based resistor; $\mathrm{I}_{\mathrm{r}} \mathrm{O}_{2}$-based resistor.

determined by the presence of the conductive grains. We shall instead proceed to the analysis of electron diffusion in the absence of applied fields and shall relate it to the mobility $\mu$ by means of the appropriate Einstein relation.

The probability per unit time for an electron in a localized state at $\vec{R}_{\mathbf{i}}$ with energy $E_{\mathbf{i}}$ to jump to a localized state in $\vec{R}_{f}$ with energy $E_{f}$ has been taken ${ }^{2}$ to be equal to

$P_{s s}(i, f)= \begin{cases}\nu_{0} \mathrm{e}^{-2 \alpha R_{i f}}\left(1-f\left(E_{f}\right)\right) & E_{f}<E_{i} \\ \nu_{0} e^{-2 \alpha R_{i f}-\left(E_{f}-E_{j}\right) / K T}\left(1-f\left(E_{f}\right)\right) & E_{f}>E_{i}\end{cases}$

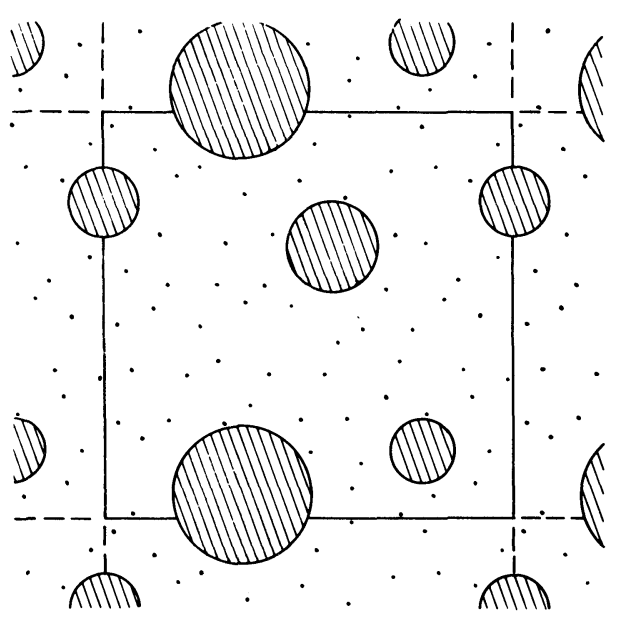

FIGURE 2 Model thick-film resistor. The basic cube generated at random is repeated periodically to simulate an infinite "homogeneous" system. 
where $f(E)$ is the Fermi distribution function, $R_{\text {if }}$ is the distance between the states, $\alpha$ is the coefficient of exponential decay of the localized states, $\nu_{0}$ is some constant which depends on properties of the material, $K$ and $T$ are the Boltzmann constant and the absolute temperature, respectively.

The analogous jump rate from a localized state to a metallic grain is obtained by assuming the above expression in Eq. (1) for a single final state inside the grain and integrating over all possible states in the grain. In the case of a jump from a grain to a localized state, we have to perform an average over all possible initial states in the grain and finally, for a grain to grain jump, both the average over the initial states and the sum over the final states must be performed. When grains are involved, the distances are taken from their borders.

For an electron in a localized state or in a grain the total probability $\tau^{-1}$ (i) per unit time of leaving its position, $i$, is given by the sum of the probabilities of jumping to any other localized state or grain:

$\tau^{-1}(\mathrm{i})=\sum_{\mathrm{f}} \mathrm{P}(\mathrm{i}, \mathrm{f})$

\section{EINSTEIN RELATION}

In order to derive the Einstein relation for the physical system under investigation, let us consider the case of a piece of material with an applied electric field $\mathrm{F}$ in open circuit conditions, when drift and diffusion currents cancel each other:

$\mathrm{n}(\mathrm{x}) \mu \mathrm{F}=\mathrm{D} \frac{\partial \mathrm{n}}{\partial \mathrm{x}}$

where $n(x)$ is the carrier density, a function of the coordinate $x$ along the direction $F$, and $D$ is the diffusion constant: to obtain $n(x)$ we have to sum the distribution function $f$ over all possible localized states and all states in metallic grains:

$n(x)=\delta_{s} \int_{E_{1}}^{E_{2}} d f(E) d E+\delta_{g} \int_{E_{c}}^{\infty} N(E) f(E) d E$

where $\delta_{\mathrm{s}}$ and $\delta_{\mathrm{g}}$ are the volume concentrations of glass and metal in the TFR; $\mathrm{d}$ and N(E) are the density of states per unit volume and unit energy in the glass and in the metal, and $\mathrm{E}_{\mathrm{c}}$ is the bottom of the conduction band in the metal. The $\mathrm{x}$ dependence of $\mathrm{n}$ comes from $E_{1}, E_{2}$ and $E_{c}$ which follow the potential energy of the thick film which varies as $\mathrm{F} \mathrm{x}$, while the Fermi level $\mathrm{E}_{\mathrm{F}}$ is constant. From Eq. (4) we may calculate $\partial \mathrm{n} / \partial \mathrm{x}$ which, when inserted in Eq. (3) yields the Einstein relation:

$\mu=\frac{\mathrm{eD}}{\mathrm{KT}} \mathrm{C}$

$C=\frac{\delta_{s} d\left\{\frac{1}{e^{\left(E_{2}-E_{F}\right) / K T}+1}-\frac{1}{e^{\left(E_{1}-E_{F}\right) / K T}+1}\right\}-\frac{1}{2} \delta_{g} B \sqrt{K T} F_{-1 / 2}\left(\frac{E_{F}}{K T}\right)}{\delta_{s} d\left\{\frac{E_{2}-E_{1}}{K T}+\ln \frac{e^{\left(E_{1}-E_{F}\right) / K T}+1}{e^{\left(E_{2}-E_{F}\right) / K T}+1}\right\}+\delta_{g} B \sqrt{K T} F_{1 / 2}\left(\frac{E_{F}}{K T}\right)}$ 
where the free electron model is assumed for the metal, $B=1 /\left(2 \pi^{2}\right)\left(2 \mathrm{~m} / \hbar^{2}\right)^{3 / 2}$ and $F_{k}(y)$ is the Fermi-Dirac integral.

When only metal is present Eq. (6) reduces to the well known ${ }^{3}$ result

$C=\frac{1}{2} F_{-1 / 2}\left(\frac{E_{F}}{\overline{K T}}\right) / F_{1 / 2}\left(\frac{E_{F}}{\overline{K T}}\right)$

When, on the contrary, only localized states are present, Eq. (6) yields

$$
C=\frac{\frac{1}{e^{\left(E_{2}-E_{F}\right) / K T}+1}-\frac{1}{e^{\left(E_{1}-E_{F}\right) / K T}+1}}{\frac{E_{2}-E_{1}}{K T}+\ln \frac{e^{\left(E_{1}-E_{F}\right) / K T}+1}{e^{\left(E_{2}-E_{F}\right) / K T}+1}}
$$

Figure 3 shows the coefficients $C$ of the Einstein relation for the physical parameters used in the present calculations (Section 5). The continuos curve indicates the classical Einstein relation $\mu=\mathrm{eD} / \mathrm{KT}$ or $\mathrm{C}=1$, valid for classical statistics (nondegenerate semiconductors). For metals (broken line) at low temperatures, $T$ must be substituted by the Fermi temperature $T_{F}$ so that $C$ becomes proportional to $T$, while at higher temperatures the Einstein relation tends to the classical results. In the case of only localized states (dotted line) the high-temperature limit is reached already when KT is larger than the energy spread $E_{2}-E_{1}$ but this limit corresponds to $C=1 / 2$ since the density of states is assumed constant.

When as in our case, both metal and localized states are present (dot-dashed line), the resulting $\mathrm{C}$ is intermediate between the two cases seen above.

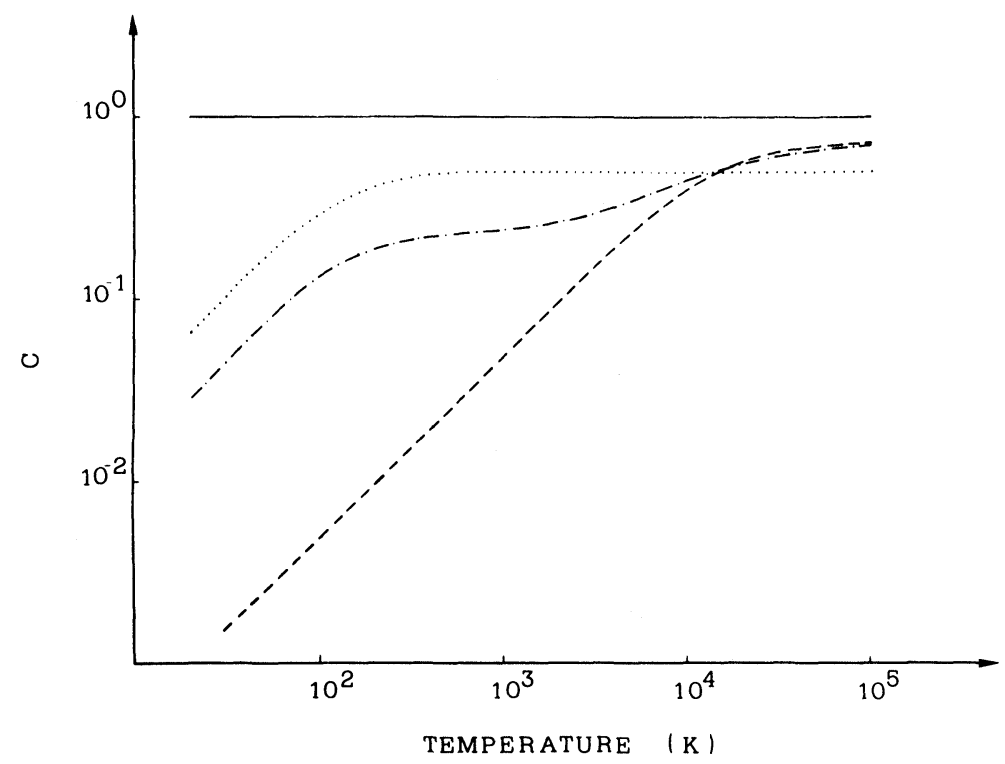

FIGURE 3 Einstein-relation coefficient $\mathrm{C}$ defined by Eq. (4) for the case of classical statistics (continuous line), metal (broken line, Eq. (7)), only localized states (dotted line, Eq. (8)), and the mixed case of TFR (dot-dashed line, Eq. (6)). The physical parameters are those indicated in Section 5. 


\section{THE MONTE CARLO PROCEDURE}

\subsection{Generation of thick films.}

A cubic piece of material is "generated" at the beginning of the simulation, whose block diagram is shown in Figure 4. Some input parameters must be given: the side L of the cube, the density $\mathrm{d}$ of localized states, the relative volume $\delta_{\mathrm{g}}$ of metal in the thick film, the maximum and minimum radii $r_{1}$ and $r_{2}$ for the grains, the maximum and minimum energy for the localized states $\mathrm{E}_{1}$ and $\mathrm{E}_{2}$, and the Fermi level $\mathrm{E}_{\mathrm{F}}$ of the metal.

\subsection{Transport.}

A number of electrons are generated at the beginning at random in localized states or in metallic states according to the probability $f(E)$ of any metallic or localized state to be

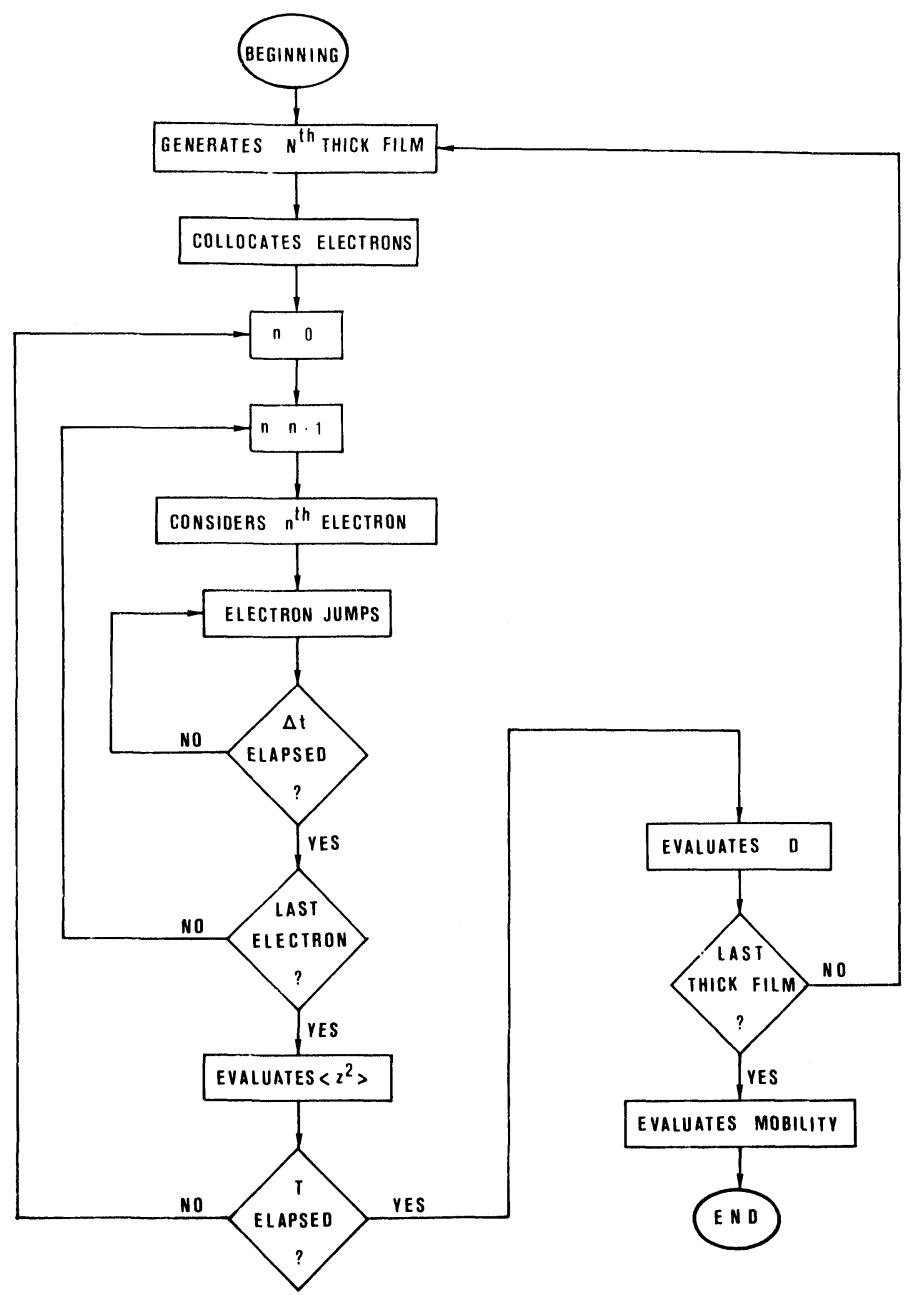

FIGURE 4 Block diagram of the computer simulation for TFR conductivity (see text). 
occupied. For each of them successive jumps are generated according to the probabilities of jump discussed in Section 2. If $\mathrm{r}$ is a random number equally distributed between 0 and 1 , the time of permanence in the state $i$ is taken to be equal to ${ }^{4}$

$$
\delta \mathrm{t}_{\mathrm{r}}=-\tau(\mathrm{i}) \ln \mathrm{r}
$$

The state of arrival is obtained by means of another random number which chooses among all possible final states according to their relative probabilities described in Section 2. The procedure is similar to what is used for high-field transport Monte Carlo simulations in semiconductors. ${ }^{4}$

In order to simulate an infinite, macroscopically homogeneous, system the cube is repeated periodically. The simulation is performed independently for all electrons and their displacement $\left(\mathrm{x}_{1}, \mathrm{x}_{2}, \mathrm{x}_{3}\right)$ from the initial position os recorded at fixed time intervals $\Delta t$ up to a total time $T$. From the simulation $\left\langle x_{i}^{2}\right\rangle$ shows a linear dependence upon time, $t$, as predicted by the diffusion theory, and the diffusion constant along a direction $x_{i}$ is then obtained by means of the equation ${ }^{5}$

$\mathrm{D}=\frac{1}{2} \frac{\mathrm{d}}{\mathrm{dt}}\left\langle\mathrm{x}_{\mathrm{i}}^{2}\right\rangle$

The diffusion constant for this simulation is obtained by averaging over the three directions. The whole process is repeated several times with a new generation of a thick film each time in order to avoid the dependence upon the particular thick film generated, and the statistical uncertainty is obtained as the variance of the various results found. From D, the mobility is obtained by applying the Einstein relation discussed above. Since the electrons, at the beginning, are generated according to their distribution, including the deep states in the Fermi sea of grains, the number of carriers to be used in the conductivity is constant, equal to the total number of electrons, so that the mobility obtained is proportional to the conductivity.

\section{RESULTS AND DISCUSSION}

Figure 5 shows the results obtained for a TFR characterized by the following physical parameters $E_{1}=E_{F}-300 \mathrm{~K}, E_{2}=E_{F}+300 \mathrm{~K}, E_{F}=30000 \mathrm{~K}, d=2.3 \times 10^{20} / \mathrm{cm}^{3}$, $\delta_{\mathrm{g}}=1.8 \times 10^{-2}, \mathrm{~L}=136 \AA, \mathrm{r}_{1}=5 \AA, \mathrm{r}_{2}=20 \AA, \nu_{0}=1 \times 10^{12}$ :

For comparison Figure 6 shows the result obtained with only localized states with the same density and energy distribution as in Figure 5.

In all cases we have a diffusion coefficient rising with temperature $T$ since a higher $T$ increases the jumping probabilities. When only localized states are present (but $E_{F}$ is kept constant), the factor $\mathrm{C}$ levels off at temperatures higher than the spread of energy levels; at the same temperatures $\mathrm{D}$ too tends to be independent of temperature. The resulting mobility (and conductivity) decreases with temperature because of the factor $\mathrm{T}^{-1}$ in the classical Einstein relation. This fact reflects the tendency of temperature to destroy an ordered response to an applied external force: when jumps are highly probable the influence of an external field which favours jumps in a given direction is less effective.

When metal grains are added many electrons lie deep in the Fermi sea, and the effect of temperature rising is sensible up to higher values of $T$ so that the conductivity keeps increasing with $\mathrm{T}$ unless the density of localized states in the glass is sufficiently high and the metal concentration sufficiently low, in which case the falling of $\mu(T)$ due to the localized states is preserved, even though made weaker, as shown in Figure 5. As a by- 


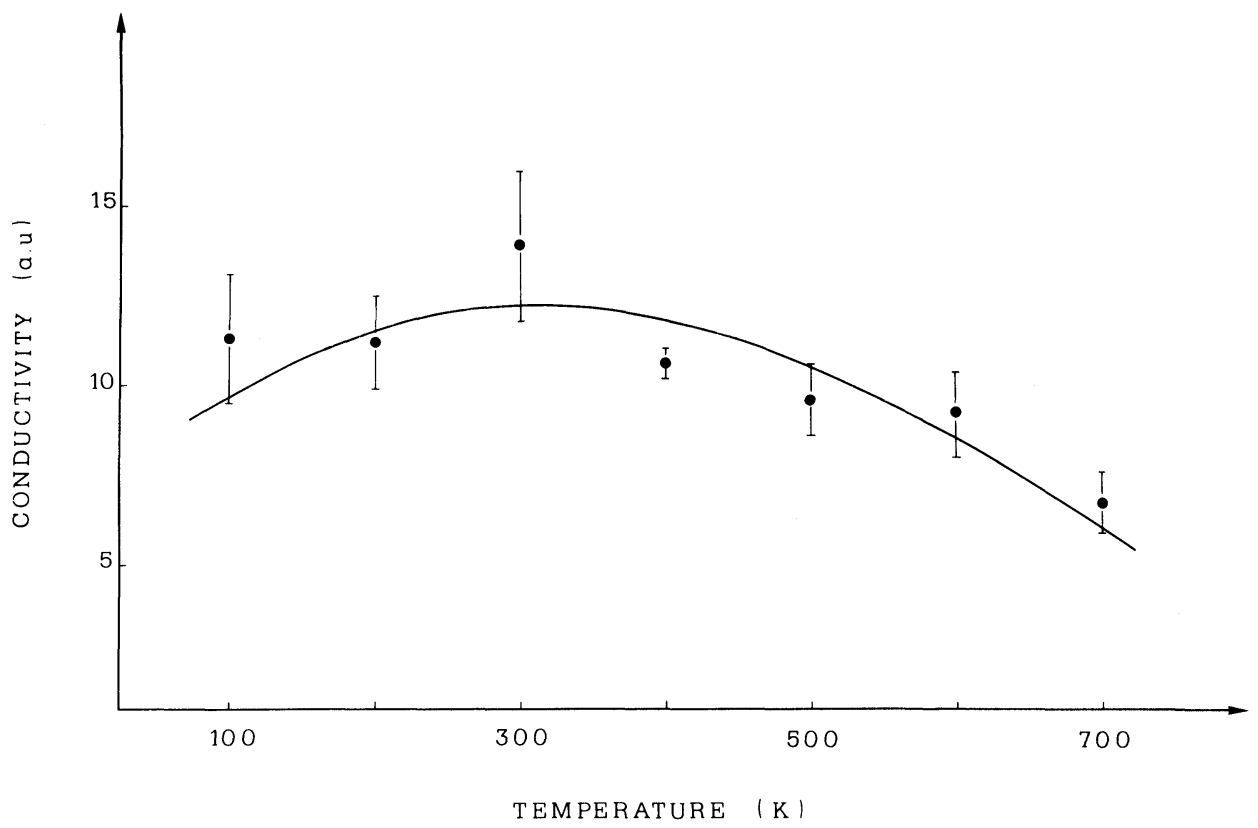

FIGURE 5 Conductivity of a model TFR obtained by means of the simulation with the physical parameters indicated in the text.

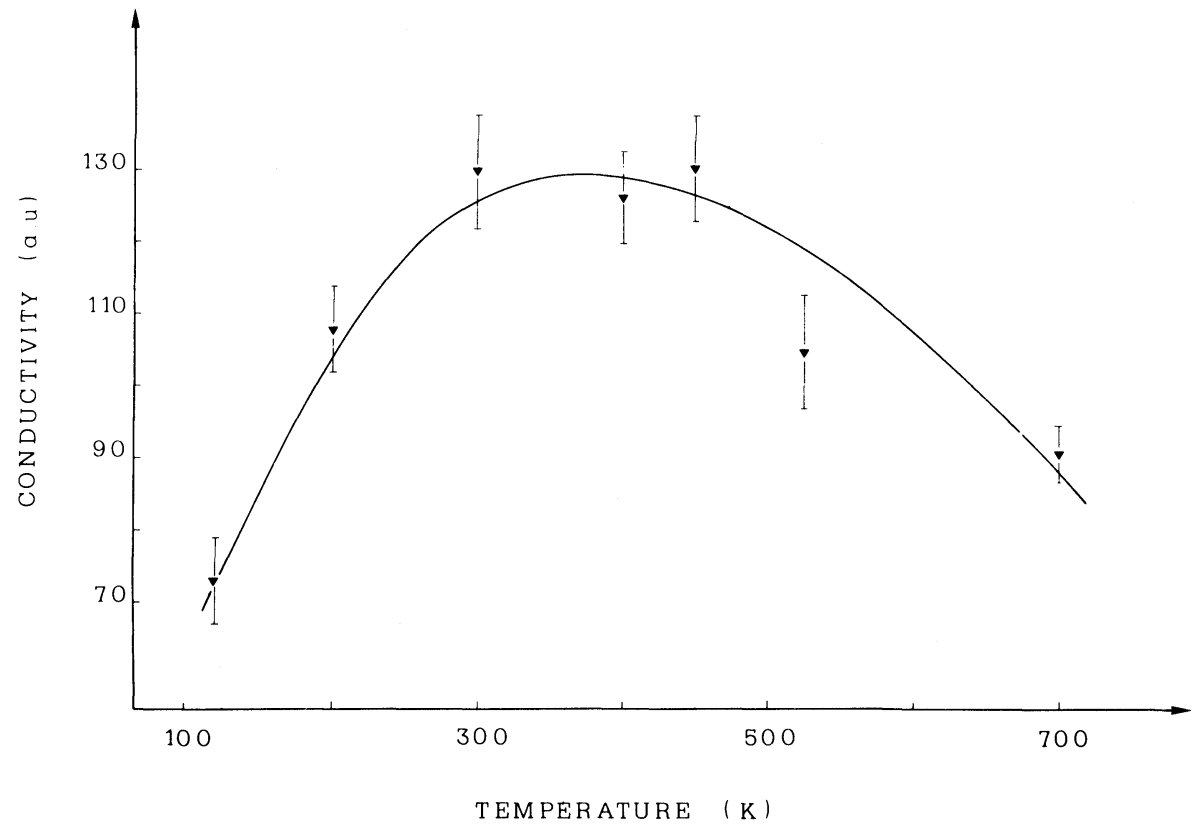

FIGURE 6 Conductivity obtained by means of the simulation of a variable-range hopping system corresponding to the case of Figure 5 without metallic grains. 
product of the simulation it was possible to confirm some percolative character of the conductivity ${ }^{6}$ indicated by privileged paths followed by the simulated electrons.

The results discussed above need to be confirmed by more realistic models, more accurate computations (which require very large computer times) and eventually it will be possible to compare them with experimental results, when enough information is available about the actual physical parameters which characterize real thick films.

Our preliminary results seem to indicate, however, that (i) a simulative approach is useful to free the theory of electrical properties of TFRs from undesirable analytical approximations, and (ii) a simple model of TFR with only metallic grains and localized states in the glass may account, under certain conditions, for the maximum of conductivity without having to make use of more sophisticated properties of TFRs.

\section{REFERENCES}

1. M. Prudenziati, Proc. European Hybrid Microelectronics Conf., 1-10 (1981).

2. V. Ambegaokar, B.I. Halperin, J.S. Langer, Phys. Rev. B4, 2612 (1971).

3. P.T. Landsberg, Proc. Roy. Soc. A213, 226 (1952).

4. W. Fawcett, D.A. Boardman, S. Swain, J. Phys. Chem. Solids 31, (1963).

5. A. Alberigi Quaranta, V. Borsari, C. Jacoboni, Appl. Phys. Lett. 22, 103 (1973).

6. F. Forlani, M. Prudenziati, ElectroComp. Sci. Technol. 3, 77 (1976). 

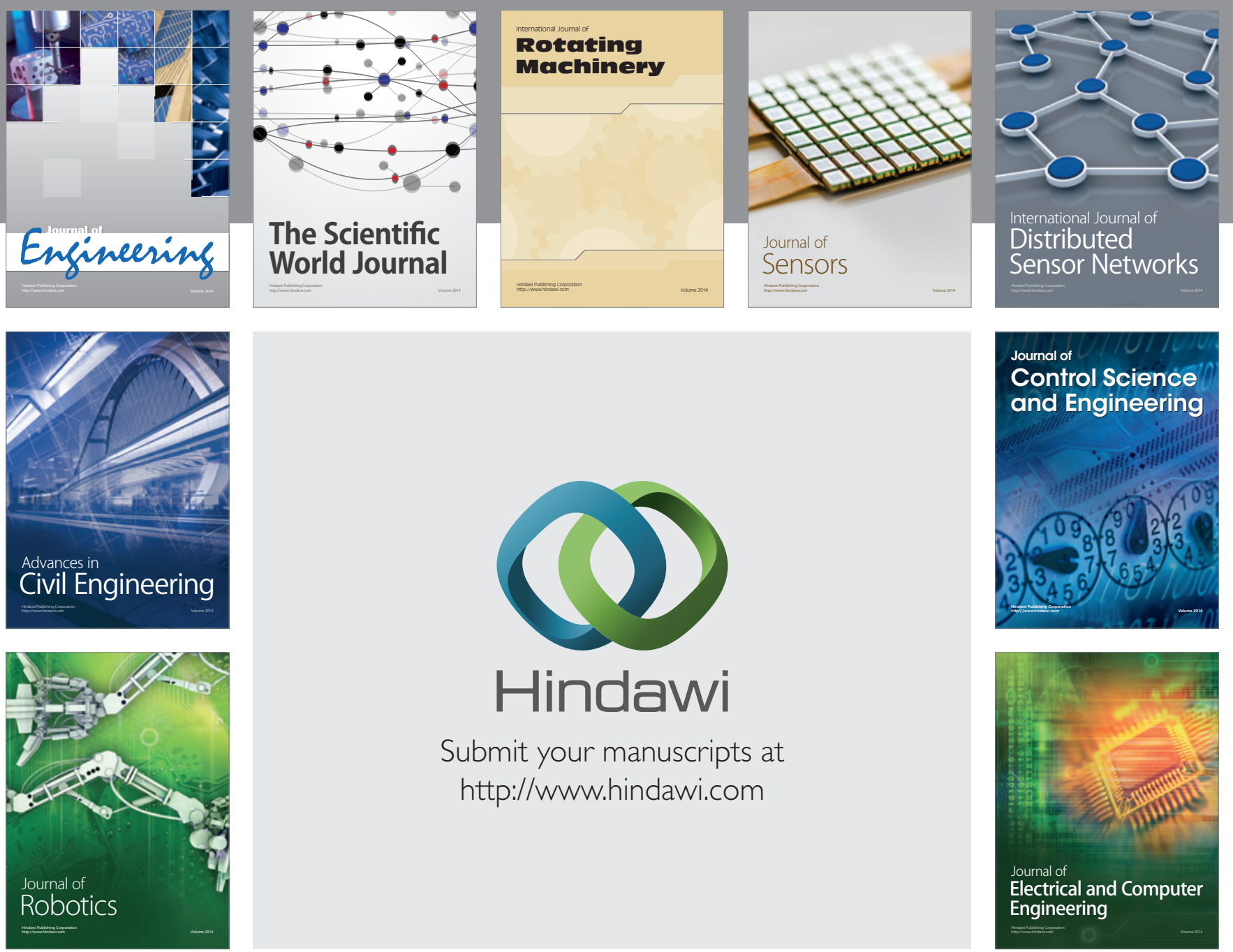

Submit your manuscripts at

http://www.hindawi.com
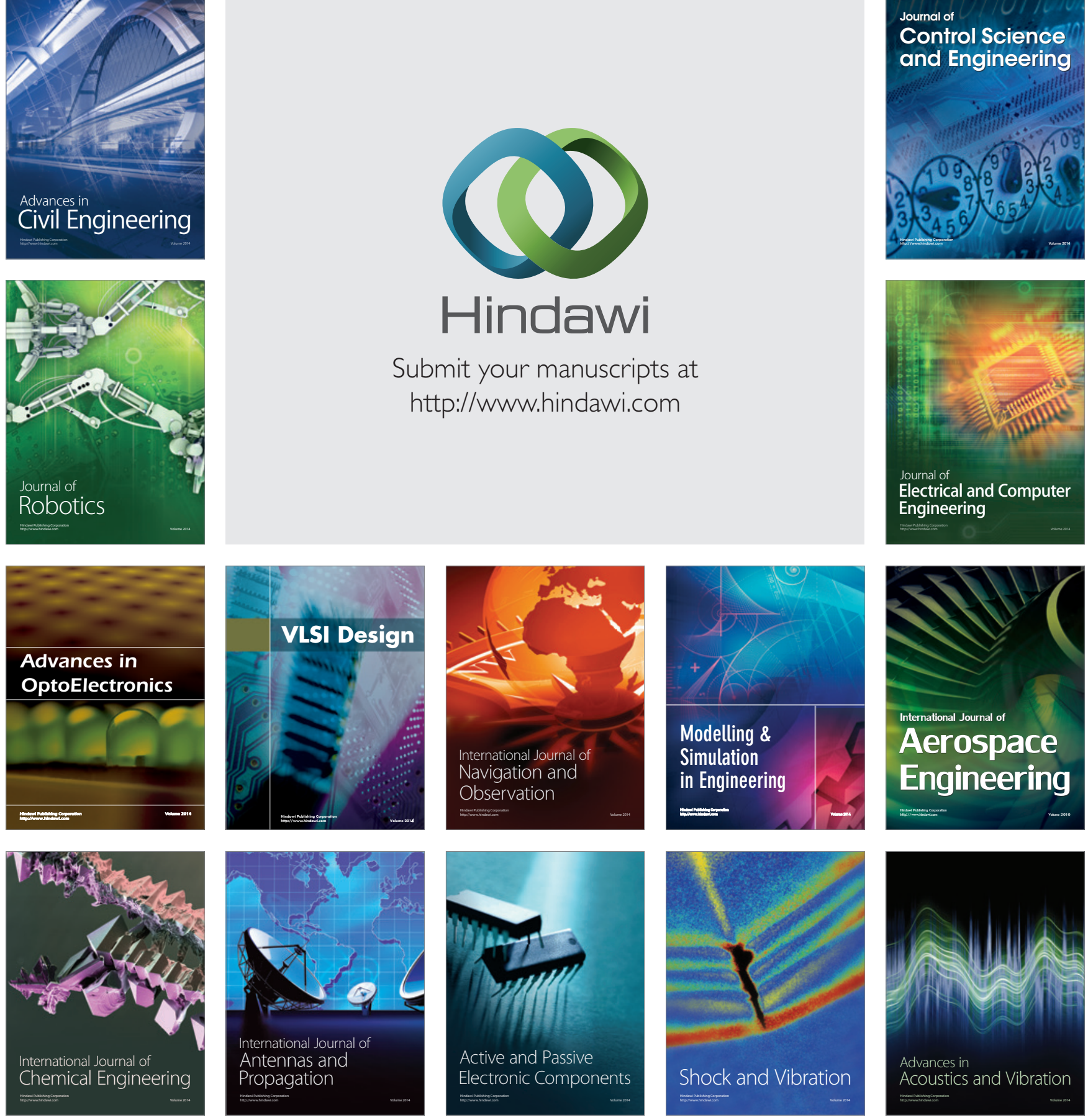